\title{
Solitary Myofibroma of the Skull Presenting Outside Infancy
}

\author{
Ralph Rahme, Gerard Abadjian, Elie Samaha
}

Can. J. Neurol. Sci. 2008; 35: 375-377

Solitary myofibromas involving the skull have been rarely reported in the literature, ${ }^{1}$ and very few cases have presented outside infancy. ${ }^{1-5}$ Such lesions may mimic other osteolytic skull lesions, particularly eosinophilic granuloma. ${ }^{1,4}$ In this report, we describe the case of a six-year-old boy presenting with a solitary myofibroma of the skull.

\section{Case Report}

A previously healthy six-year-old boy presented with painless swelling in the left frontal region that had been slowly progressing in size over the past four months. The patient denied any accompanying systemic or neurological symptoms. On exam, a 2-cm firm lesion was palpable in the left parasagittal frontal region. The lesion was not tender to palpation and there was no surrounding inflammatory reaction. Neurological exam was unremarkable. A thorough general physical examination did not reveal any other similar lesions or lumps in the patient's body. Computed tomography scan of the head revealed a heterogeneous hypodense osteolytic lesion extending across the full thickness of the skull with possible involvement of the underlying dura. The lesion did not exhibit a trabecular pattern and had irregular, smooth, nonsclerotic margins (Figure 1).

Differential diagnosis at this time included eosinophilic granuloma, hemangioma, and epidermoid or dermoid cyst. After discussion of the management options with the parents, it was decided to surgically excise the lesion. The patient was taken to the operating room where complete en bloc resection was performed under general anesthesia. The lesion seemed to infiltrate the outer leaflet of the underlying dura mater which was also resected. The inner dural leaflet and superior sagittal sinus were not involved and were left intact. Finally, cranioplasty was performed using split-thickness skull autograft.

Pathological examination of the lesion revealed an ill-defined fibrous nodule with proliferating fusiform cells arranged in fascicles around numerous distended vascular spaces in a hemangiopericytomatous pattern. Immunohistochemical staining

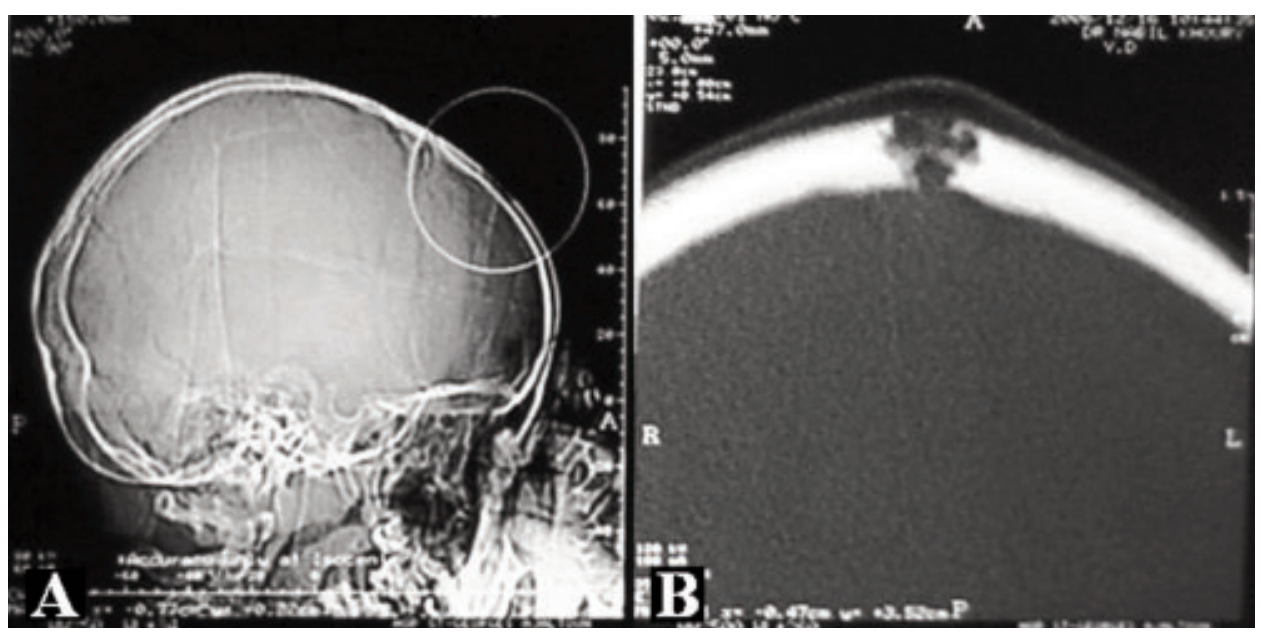

Figure 1. A and B, Plain head CT reveals a heterogeneous osteolytic skull lesion involving the full thickness of the skull. Infiltration of the underlying dura mater cannot be excluded. The lesion does not exhibit any trabecular pattern and has irregular, smooth, nonsclerotic margins.

\footnotetext{
From the Departments of Neurosurgery (RR, ES), Pathology (GA), Saint-Joseph University and Hôtel-Dieu de France, Beirut, Lebanon.

Received August 7, 2007. Final Revisions Submitted January 25, 2008.

Reprint requests to: Elie Samaha, Department of Neurosurgery, Hôtel-Dieu de France, Ashrafieh, Beirut, Lebanon.
} 
using the biotin-avidin technique demonstrated cell positivity for smooth muscle actin (clone 1A4; source BioGenex, San Ramon, CA; dilution 1:100) and negativity for EMA (E29; Dako, Glostrup, Denmark; 1:100), S-100 (polyclonal; Dako; 1:3000), desmin (D33; Dako; 1:200), and CD34 (QBEnd10; Dako; 1:50). There was no atypia or necrosis and mitotic activity (Ki-67) was low (MIB-1; Dako; 1:50). Examination of the dural leaflet showed no evidence of infiltration by the tumor. Pathology was consistent with a myofibroma (Figure 2).

The patient had an uneventful post-operative course and was discharged home the next morning. He has been completely asymptomatic since then. At his last follow-up 12 months later, there was no evidence of local or systemic recurrence of the lesion.

\section{DISCUSSION}

Myofibroma was initially described in 1954 by Stout as "congenital generalized fibromatosis". 6 Subsequently, two distinct forms of this disease have been identified: the multicentric variant or myofibromatosis and the more common solitary form. ${ }^{7}$ While solitary lesions generally follow an entirely benign course, multicentric lesions involving the viscera may ultimately be fatal owing to acute massive cardiopulmonary failure, hemorrhage or diffuse myofibromatous infiltration and obstruction of the gastrointestinal tract. ${ }^{8}$

Myofibroma is a benign fibrous tumor seen almost exclusively in infants and young children. This tumor can arise in the soft tissues, bones, or viscera. The head and neck are the most common location, followed by the trunk and extremities. ${ }^{8}$ Although rare, myofibroma is the most common fibrous tumor of infancy. ${ }^{9}$

Histologically, myofibroma has two distinctive features. First, cells are intermediate in appearance between fibroblasts and smooth muscle cells and are arranged in short bundles or fascicles. Second, there is a prominent central vascular arrangement with a hemangiopericytomatous pattern. ${ }^{1}$ Although aggressive histological features such as a relatively high mitotic rate, nuclear atypia, areas of necrosis, and subendothelial intravascular tumor growth may be present, these have no bearing on the clinical outcome. ${ }^{10}$ Immunocytochemical staining of tumor cells reveals vimentin and alpha-smooth muscle actin reactivity, with lack of immunoreactivity to $\mathrm{S}-100$ protein, epithelial membrane antigen, keratin, and desmin. ${ }^{10}$ Ultrastructural examination shows myofibroblastic differentiation with prominent dilated rough endoplasmic reticulum, longitudinal filaments with dense bodies, and focal basal lamina. ${ }^{10}$

Cytogenetic analyses of myofibroma have shown nonspecific findings with chromosome 8 abnormalities and, although the vast majority of these lesions occur sporadically, a few reports of familial myofibromatosis have implicated an autosomal dominant inheritance pattern. ${ }^{10}$

The pathological differential diagnosis of myofibroma should include other pediatric fibroblastic and myofibroblastic tumors such as infantile fibrosarcoma, solitary fibrous tumor, and desmoid tumor. Among these, infantile fibrosarcoma may be the one most difficult to differentiate from myofibroma. ${ }^{10}$ Malignant histopathological features such as high cellularity in a herringbone pattern, nuclear hyperchromasia, necrosis, and

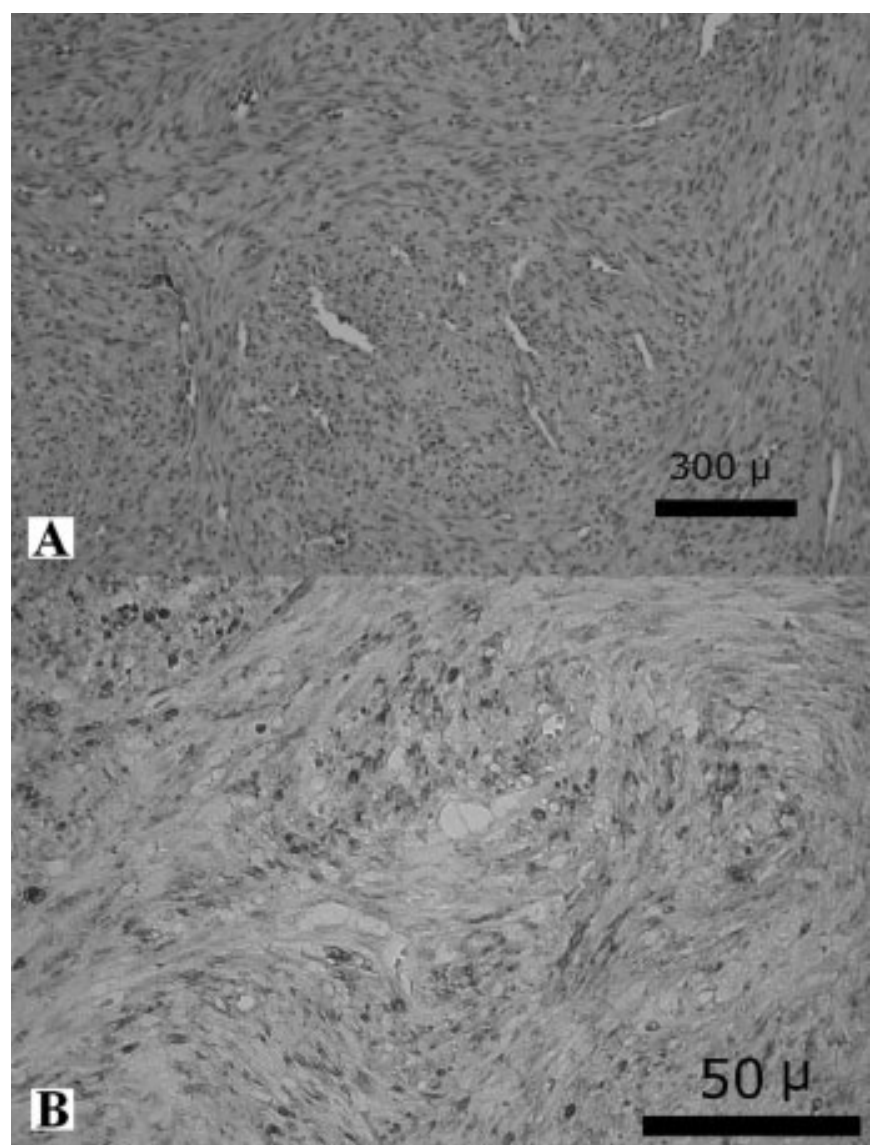

Figure 2. A) Photomicrograph showing proliferating myofibroblastic cells with low mitotic activity and no atypia, arranged in fascicles around numerous distended vascular spaces in a hemangiopericytomatous pattern $(H \& E)$. B) Immunohistochemical staining demonstrates positivity for smooth muscle actin.

cystic degeneration may help distinguish infantile fibrosarcoma from myofibroma. ${ }^{10}$ Moreover, characteristic ultrastructural features such as lack of extracellular banded collagen, rare to absent basal lamina, branching and markedly dilated rough endoplasmic reticulum, and irregular nuclear membranes may also orient the diagnosis toward infantile fibrosarcoma. ${ }^{10}$ Most importantly, myofibroma lacks the tumor-defining translocation between chromosomes 12 and 15 (ETV6-NTRK3) found in infantile fibrosarcoma. In particularly difficult cases, searching for this translocation should be performed to eliminate infantile fibrosarcoma as a diagnostic consideration. ${ }^{10}$

Among solitary osseous myofibromas, those involving the skull are most common. ${ }^{11}$ Such lesions have been rarely reported in the literature, ${ }^{1}$ and only very few cases have presented outside infancy. ${ }^{1-5}$ These tumors typically present as osteolytic hypodense lesions that enhance strongly with intravenous contrast. ${ }^{3,12}$ They may be difficult to differentiate from other osteolytic lesions affecting the skull, particularly eosinophilic 
granuloma. ${ }^{1,4}$ These lesions usually do not violate the arachnoidal or galeal layers and do not infiltrate the brain, but instead cause local compression and displacement. ${ }^{12}$ Due to their slow-growing nature, neurological symptoms are generally absent, even with very large tumors. ${ }^{1}$ Surgical excision of solitary skull myofibroma is usually curative with no tumor recurrence being reported following total resection. ${ }^{1,3}$

\section{CONCLUSION}

Although very rare, solitary myofibroma should always be considered in the differential diagnosis of osteolytic skull tumors in infants, older children, and adults. Total resection of this lesion is usually curative.

\section{REFERENCES}

1. Bodkin PA, Choksey MS, Fagan J. Solitary calvarial myofibroma presenting in adolescence. Br J Neurosurg. 2005; 19:420-4.

2. Mouri W, Kayama T, Sakurada K, Sato S, Saino M, Nakazato Y. A case of giant skull myofibroma occupying left anterior cranial fossa [in Japanese]. No To Shinkei. 2000; 52:821-6.

3. Tsuji M, Inagaki T, Kasai H, Yamanouchi Y, Kawamoto K, Uemura Y. Solitary myofibromatosis of the skull: a case report and review of literature. Childs Nerv Syst. 2004; 20:366-9.
4. Queralt JA, Poirier VC. Solitary infantile myofibromatosis of the skull. AJNR Am J Neuroradiol. 1995; 16:476-8.

5. Franzese CB, Carron J. Infantile myofibromatosis: unusual diagnosis in an older child. Int J Pediatr Otorhinolaryngol. 2005; 69:865-8.

6. Stout AP. Juvenile fibromatoses. Cancer. 1954; 7:953-78.

7. Kauffman SL, Stout AP. Congenital mesenchymal tumors. Cancer. $1965 ; 18: 460-76$

8. Chung EB, Enzinger FM. Infantile myofibromatosis. Cancer. 1981; 48:1807-18.

9. Wiswell TE, Davis J, Cunningham BE, Solenberger R, Thomas PJ. Infantile myofibromatosis: the most common fibrous tumor of infancy. J Pediatr Surg. 1988; 23:315-8.

10. Hicks J, Mierau G. The spectrum of pediatric fibroblastic and myofibroblastic tumors. Ultrastruct Pathol. 2004; 28:265-81.

11. Inwards CY, Unni KK, Beabout JW, Shives TC. Solitary congenital fibromatosis (infantile myofibromatosis) of bone. Am J Surg Pathol. 1991; 15:935-41.

12. Rutigliano MJ, Pollack IF, Ahdab-Barmada M, Pang D, Albright AL. Intracranial infantile myofibromatosis. J Neurosurg. 1994; 81:539-43. 